(2) Open Access Full Text Article

\title{
Competing neurobehavioral decision systems and the neuroeconomics of craving in opioid addiction
}

\author{
This article was published in the following Dove Press journal: \\ Neuroscience and Neuroeconomics \\ 8 October 2014 \\ Number of times this article has been viewed
}

\author{
Michael J Sofis' \\ David P Jarmolowicz' \\ Laura E Martin² \\ 'University of Kansas, ${ }^{2}$ University \\ of Kansas Medical Center, \\ Lawrence, KS, USA
}

Correspondence: David P Jarmolowicz Center for Applied Neuroeconomics, Department of Applied Behavioral Science, University of Kansas, 400I Dole Developmental Center, 1000 Sunnyside Ave, Lawrence, KS 66045, USA Email dpj@ku.edu

\begin{abstract}
Craving is typically thought of as a classically conditioned response characterized by an elevated mesolimbic dopamine response to drug-related stimuli. Although this definition has spurred considerable research, the clinical impact of the research conducted has been less robust. The current review takes a more contemporary approach by conceptualizing craving as the breakdown of executive function and relative strengthening of the limbic system, occurring in the presence of conditioned cues, leading to a maladaptive craving response (ie, an increased likelihood of drug consumption). Working from this framework, the present review focuses on four issues in drug craving research: pivotal findings and limitations of cue-reactivity and neurocognitive tasks; two main processes of craving that include self-control and reward-based explanations; integration of neuroeconomic approaches to craving; and the theoretical implications and future directions of drug craving research.
\end{abstract}

Keywords: craving, competing decision systems, executive function, loss of control, substance abuse

\section{Introduction}

"Craving" is the breakdown of executive function and relative strengthening of the limbic system, occurring in the presence of conditioned cues, leading to a maladaptive craving response (ie, an increased likelihood of drug consumption). ${ }^{1-3}$ The utility of this definition springs from its direct focus on the relationship between craving responses and problematic drug consumption. From this viewpoint, neurobehavioral markers of craving are less relevant if they do not reliably predict actual drug consumption. Craving, although widely studied, has not been consistently successful as a treatment target. ${ }^{4,5}$

Substance-specific differences in craving appear to be less relevant than alternative factors such as treatment status, ${ }^{6}$ mode of cue, and the match between the cue presented and the characteristics of the cues present in the individual's drug use settings. ${ }^{7}$ Drug-specific differences in craving are inconsistently observed. The disparity between findings could be due to other factors, such as participant characteristics (eg, age), mode of physiological measurement, and type of self-reported craving assessment. ${ }^{8}$ The majority of reviews on drug craving are not commodity-specific but instead target factors such as common regions of activation, ${ }^{9}$ theoretical approaches, ${ }^{10}$ or treatment status. ${ }^{6}$ Currently, the exploration of substance-specific differences appears to be less relevant than other methodological considerations, and this review therefore focuses on craving as manifest across many drugs of abuse. 
The practical utility of craving has been questioned due to inconsistent translation from theory to treatment. ${ }^{9}, 10$ The most prevalent model of craving, the cue-reactivity paradigm, has proven crucial to better understanding of the neurobiological underpinnings of craving. In addition, the cue-reactivity paradigm has provided a framework conducive to measuring physiological responses to cues, typically conceptualized as a stimulus-response relationship. The theoretical background of the cue-reactivity paradigm, however, has several limitations that could be responsible for ineffective translation to treatment findings. ${ }^{11}$ The most relevant of these limitations is the dearth of research that integrates advancements from the findings of neuroscience, cognitive psychology, and behavioral economics. The main goal of the present review is to communicate a conceptual and methodological framework of neuroeconomics as it applies to the study of drug craving. The present paper therefore introduces how neuroeconomic tools and methods can provide such a synthesis and address many existing limitations of craving research. Working from this framework, the present review focuses on five issues in drug craving research: pivotal findings and limitations of cue-reactivity and neurocognitive task paradigms; the two resultant processes of craving that include self-control and reward-based explanations; limitations of existing neuroeconomic approaches to craving and how emerging neuroeconomic approaches can fill these gaps; and lastly, theoretical implications and future directions of drug-craving research.

\section{Cue-reactivity and neurocognitive task paradigms: findings and limitations}

Drug craving is typically defined as a classically conditioned response characterized by an elevated mesolimbic dopamine response to drug-related stimuli. ${ }^{8,12-14}$ Working from this framework, cue-elicited and/or cue-reactivity paradigms are predominantly used to evaluate drug craving. ${ }^{6,11}$ In cueelicited craving studies, subjects' physiological responses (eg, blood oxygen levels via functional magnetic resonance imaging [fMRI]; electroencephalography event-related potentials) and self-reports of craving (commonly referred to as subjective craving) are typically assessed before and after the presentation of neutral and/or drug stimuli.

Cue-elicited craving studies have provided a valuable insight into important relationships between neural correlates of drug cues and regional and systems-level neural networks. For example, the accumulating literature has identified that four of the most commonly cited loci of activation during cue-elicited tasks in non-treatment-seeking individuals are the dorsal lateral prefrontal cortex (dlPFC; goal seeking), amygdala (conditioned drug seeking), anterior cingulate cortex (ACC; affective processing), and orbitofrontal cortex (OFC; expectancy). ${ }^{6}$ More recently, the insula has been identified as an additional region commonly activated during craving. ${ }^{15,16}$ Brain activity in these regions has also been shown to predict relapse. ${ }^{17}$

Comparisons of patterns of neural activation during neurocognitive and cue-elicited tasks have isolated the brain regions associated with craving (eg, amygdala, ACC, dlPFC, $\mathrm{OFC}$, and insula). Relatedly, executive functioning deficits in areas such as valuing future events (delay discounting), working memory, cognitive flexibility, risk taking, and attention are consistently related to substance abuse and other impulse control disorders (eg, obesity, gambling ${ }^{18-20}$ ). Analyses of the patterns of neural activation during these neurocognitive assessments suggest that task performance reflects the relative activation in two interconnected neurobiological systems: the evolutionarily older impulsive system, which is rewarddriven and housed in limbic and prelimbic areas (eg, ACC, amygdala), and the more recently evolved executive system, which underlies planning and self-control processes and is housed in prefrontal regions (eg, dlPFC). Because of the similarity in neural regions implicated in executive function and craving, executive functioning deficits may be pivotal to understanding craving and may indicate an individual's susceptibility to addiction. ${ }^{21,22}$

The cue-reactivity paradigm has led to a better understanding of the neurobiological underpinnings of addiction; however, an over-reliance on cue-reactivity has two main limitations: an over-reliance on a classical conditioning framework and subsequently an overemphasis on physiological measurement of craving.

Much research utilizing the cue-reactivity paradigm relies heavily on the theoretical underpinnings of classical conditioning. ${ }^{8}$ Such a framework focuses almost exclusively on the neurobiological events that occur within close temporal proximity to the craving response while leaving no explanation for events that occur outside that small temporal window. This framework does not lend itself well to emerging evidence that executive system activation mediates a selfcontrol mechanism during craving. ${ }^{21,23}$ Further, it excludes decision-making processes mediated by the executive system that occur prior to the temporal window within which craving is thought to occur. Conversely, classical conditioning accounts of craving exclude what occurs between the craving response and drug consumption. More specifically, there is 
no explanation of the role of the goal-directed behavior that is likely mediated via operant learning. ${ }^{16}$ Although it is likely that respondent conditioning occurs during autonomic reward seeking (ie, drug seeking without barriers to consumption), it is equally likely that prefrontal functions such as working memory, attention, and reward valuation are being utilized to facilitate goal-directed reward seeking (ie, drug seeking in the presence of barriers). ${ }^{15,24} \mathrm{~A}$ theoretical framework that hopes to explain craving within addiction must encapsulate both of these reward-based explanations of drug seeking.

Second, the cue-reactivity paradigm focuses heavily on generalized physiological responses (eg, blood oxygenation level dependent, skin conductance, heart rate) that are not as sensitive to cue manipulations as are many subjective assessments of craving. ${ }^{8}$ Alternative factors during cue presentation are also likely to confound measurement of automatic physiological responses. ${ }^{25}$ Examples are cognitive effort related to the upcoming self-control task ${ }^{26}$ and negative affect linked with the substance user being denied the drug to consume. ${ }^{27}$ An emphasis on physiological measurement of craving is also particularly conducive to measuring the type of stimulus-response relationships typically seen in classical conditioning. These two limitations may limit methodologies used to explore what is happening before the craving response and during drug seeking behavior (ie, after the craving response). This is particularly undesirable as the utility of craving as a construct is inexorably linked with its relationship to maladaptive drug consumption. Together, the over-reliance on physiological measurement and classical conditioning in cue-elicited studies constrains conceptual and methodological efforts to understand drug craving.

The field of neuroeconomics, an integration of psychology, economics, and neuroscience, may help facilitate the interaction of cue-elicited craving with a broader array of psychological methods. Neuroeconomics stems in part from the rejection of human "rationality" in decision-making previously purported by traditional economists. ${ }^{28}$ For the sake of this review, neuroeconomics will be defined as a combination of neuroscience, psychology, and behavior economic methods that explore complex processes underlying automatic and reflective behavior through the study of decision-making under risk and uncertainty, intertemporal choice (delay discounting), and social decision-making (eg, altruism) ${ }^{29}$ Based on this definition, studies incorporating subjective craving scales (eg, Likert 1-10) in conjunction with a cue-elicited paradigm would not be considered a neuroeconomic approach. Many neuroeconomic approaches to craving, however, incorporate evidence from studies that do not incorporate neuroeconomic methods in order to better understand the underlying processes of craving.

\section{Self-control and reward processes of craving}

The interplay between reward-based (ie, impulsive system) and self-control-based (ie, executive system) processes has emerged from the neuroeconomic literature as an underlying explanation of the transition from craving to maladaptive drug consumption. ${ }^{1,2,30}$ These respective processes are likely interdependent, and the failure of one depends in large part on individual differences. ${ }^{1,21}$

Impulsive system activation during craving is closely tied to activity of the mesolimbic dopamine system. Reward-based processes are argued to underlie the progressively increased salience of drug-related cues, to the exclusion of non-drug cues. This elevated salience of drug-related cues is thought to contribute to the increased propensity of cue-induced relapse. ${ }^{31,32}$ Researchers working from this framework, often called incentive salience, ${ }^{13,31}$ posit that other contributors to relapse (eg, irrational decision-making) are a function of incentive salience processes. The mesolimbic dopamine system is largely thought to influence the reinforcing characteristics of the drug through activity in the limbic regions. ${ }^{12,33} \mathrm{As}$ previously mentioned, however, four of the most commonly cited regions of activation during cue-elicited tasks are the amygdala, OFC, ACC, and dlPFC, two of which (the OFC and dIPFC) are subcomponents of the executive system. ${ }^{34,35}$ Additionally, interactions between the mesolimbic dopamine system and executive system regions are occurring simultaneously during a craving response, suggesting a potential dynamic process. ${ }^{36}$ Thus, craving cannot be solely accounted for by appeals to the mesolimbic dopamine system. ${ }^{2,37}$

Particular brain regions activated during neuroeconomic tasks might reveal a moderating function between executive and impulsive systems. ${ }^{21}$ The ACC, for example, is related to the individual's ability to inhibit responding during neurocognitive tasks such as the Go-No-Go ${ }^{38}$ or Stop-Signal Reaction Time Task. ${ }^{39}$ Although it is unclear what accounts for differences in brain activation between similar cue-elicited methodologies, one potential explanation is the perceived availability of the drug (ie, during the experiment or a delayed period afterwards) ${ }^{6}$. In the end, evidence of executive system activation concurrent with impulsive system activation during cue-elicited tasks suggests that multiple factors contribute to craving, not just those deriving from the incentive sensitization theory. 
An alternative approach is to conceptualize much of the neural response that we associate with craving as a top-down (executive system) counter to the cue-elicited mesolimbic response. The breakdown of control by these sorts of selfcontrol networks is associated with a "loss of control" and the subjective experience of craving, ${ }^{3,23,40}$ leading to an increased likelihood of drug consumption. ${ }^{9,21}$ These sorts of self-control-based explanations of craving are not mutually exclusive from reward-based explanations. Instead, selfcontrol and reward are likely inter-related modules that, when broken down, facilitate the transition from initial drug use to dependence. ${ }^{23,41}$ "Loss of control" can be further categorized into subcomponents based on the environmental trigger, such as stress, emotion, incentive salience, pain, and/or decisionmaking. ${ }^{1}$ Support for the loss of control that takes place during drug craving comes in part from cue-elicited work using a $2 \times 2$ factorial design to explore neural correlates to drug and neutral stimuli in addition to using both substance abusers and controls. ${ }^{3,23,41}$ This methodological adjustment from only using drug stimuli alongside treatment and control groups has revealed that heroin-dependent individuals have shown lower levels of baseline activation in the executive system compared with controls when exposed to drug versus neutral stimuli. ${ }^{23,41}$ In some instances, baseline levels of executive system performance were predictive of the level of reactivity participants exhibited toward the drug-related cue. ${ }^{40}$ Interestingly, in a review of 19 cue-elicited studies across a variety of substances and cue modalities (eg, visual, tactile, video, gustatory), ten of ten studies using active drugseeking populations found significant relative activation in either the OFC or dlPFC, whereas only one of nine studies using treatment-seeking individuals found significant activation in either region. ${ }^{6}$ This further supports the link between deficits in the executive system and the craving observed in more seriously addicted individuals.

\section{A consensus model of craving}

A comprehensive model of craving must synthesize both reward (impulsive system) and self-control (executive system) processes at a behavioral and biological level. Whether the neural mechanisms that undergird craving work as top-down or bottom-up processes, however, remains unclear. ${ }^{21}$ If one process precedes and causally impacts the other, the distinction between a top-down and bottom-up process is crucial because the directionality of the process will guide treatment efforts. Specifically, if top-down processes govern craving responses, interventions that target executive processes are indicated, whereas if bottom-up processes spur craving, tamping down the impulsive system would be suggested.$^{21} \mathrm{~A}$ comprehensive model of craving must address the degree to which individual differences inform top-down or bottom-up treatment strategies. ${ }^{42}$ Ultimately, the utility of any craving model will be judged by its impact on treatment efficacy.

Craving may reflect the inter-related processes of selfcontrol and reward. ${ }^{1,21}$ Thus, multiple patterns of neural activity often undergird a single craving response. ${ }^{3,23,30}$ For example, Yang et $\mathrm{al}^{23}$ discovered that patterns of activation in an impulsive system network of the ventral tegmental area, amygdala, fusiform cortex, and precuneus were inter-related with an interconnected prefrontal system (OFC, superior frontal cortex, middle frontal cortex), which is implicated in the loss of control following a craving response. Interestingly, the authors suggest that the connections within this particular prefrontal system may be indicative of a "loss of control" as the user ignores distracting stimuli and engages in goal-directed behavior toward drug consumption. Similarly, non-human studies have demonstrated the complexity of neural patterns that underlie craving by observing that reinstatement following extinction is mediated by three separate neurotransmitter pathways, including a glutamatergic pathway connecting the prefrontal cortex to the nucleus accumbens. ${ }^{2,30,43,44}$

Intervention studies may provide guidance regarding the relative role of top-down and bottom-up processes in craving. If strengthening of the executive system through neurocognitive training decreases craving, top-down processes are suggested. ${ }^{9,30}$ For example, Volkow et $\mathrm{al}^{30}$ instructed participants to control their cocaine craving while observing drug-related cues. Using a within-subject design (baseline, pre-, and post-positron emission tomography scans), the researchers found that participants in the cognitive control condition were able to control inhibition based on an increase in activation in the right inferior frontal cortex and a decrease in activation in the nucleus accumbens. Interestingly, activation in the nucleus accumbens was negatively correlated with that in the right inferior frontal cortex when participants attempted to cognitively control cravings. The right inferior frontal cortex has been strongly associated with inhibition, ${ }^{38}$ and its relationship with the nucleus accumbens represents clinical promise for top-down cognitive interventions that impact the fronto-accumbal circuit. ${ }^{30}$ Despite the effort to determine the relative roles of top-down versus bottom-up processes in craving, it remains difficult to posit which system is a more relevant target for treating drug craving. 


\section{Integrating neuroeconomic approaches to craving}

Many approaches to craving continue to rely heavily on cue-elicited and/or associative learning-based approaches, thus hampering future empirical efforts. The use of novel conceptual and methodological approaches used by neuroeconomists may help uncover the behavioral and neurobiological mechanisms of craving. Barriers include a lack of emphasis on within-subject experimental designs, little empirical connection between cue-elicited and cognitive task paradigms, a disproportionate focus on the disrupted neural mechanisms present during addiction rather than pre-existing neural dysfunction, ${ }^{1}$ and little empirical attention devoted to goal-directed behavior that occurs between a maladaptive craving response and drug consumption. ${ }^{15}$

Studies of the neural underpinnings of craving generally use group designs comparing measures of craving before and after drug cue presentation. ${ }^{10}$ These designs typically do not include the within-subject replication that has been the basis of inference in behavioral studies ${ }^{45}$ Specifically, within-subject replication is needed in order to make inferences about the behavior of any specific individual within the study, and is a prerequisite to making strong statements about individual differences. Although this emphasis on between-subject inference may be justified due to the expense of conducting extensive within-subject replication, the loss of inferential resolution should not be ignored. The power of these sorts of within-subject analyses is well demonstrated via work on real-time neurofeedback. Neurofeedback makes reward contingent on within-subject patterns of brain activation, demonstrating one-to-one correspondence between a given subject's experience with the independent variable (ie, reward for specific patterns of neural activity) and the reported dependent variable. ${ }^{46}$ This intimate contact with the independent variable may be highly effective treatment for maladaptive craving. For example, Canterberry et $\mathrm{al}^{47}$ performed three real-time fMRI sessions and found that subjective craving scores and activation in the ACC decreased with each consecutive session.

Despite the potential importance of doing so, few studies have examined cognitive performance and cue-elicited craving within the same group of subjects. Such data would allow researchers to examine the relationships between cognitive performance and craving that mediate individual differences. Cognitive function and cue-elicited craving researchers often reference each other's work, but the lack of withinsubject comparisons makes unequivocal statements about how these patterns of responding relate to other variables difficult (eg, characteristics of population, drug, type of neural measurement). Volkow et al, ${ }^{30}$ for example, found that when subjects responded to instructions to inhibit craving, activation of metabolic activity in the right inferior frontal cortex increased, whereas activity decreased in the right OFC and right accumbens. One self-proclaimed limitation of the study was a failure to demonstrate a relationship between neural activation and subjective cravings. The authors posited that the lack of a relationship may have been due to the poor sensitivity of the craving measure used (ie, a Likert scale about subjective craving) and could be remedied by asking participants how much they would be willing to pay for the drug. This measure was hypothesized to correlate with the relationship between the nucleus accumbens and the medial OFC. This task would be similar to the hypothetical purchase task used in behavioral economic studies. ${ }^{48,49}$ Interestingly, MacKillop et a $\mathrm{l}^{49}$ have recently found this measure to be a sensitive measure of cue-elicited cigarette craving. Incorporating behavioral measures into neurobiological studies in this example not only provides the potential for improved validity in place of subjective measurement of craving, but also makes a plethora of research available for cross-referencing. ${ }^{50}$ Moreover, neuroeconomic analyses of reward characteristics may further identify important differences between clinical populations (eg, drug users) and non-clinical populations..$^{18}$ Another example comes from Verdejo-Garcia et al, ${ }^{51}$ who examined response inhibition (ie, Stroop performance) before and after a drug-related cue in drug users and community controls. They found that individuals' compulsivity scores on the Obsessive Compulsive Drug Use Scale ${ }^{52}$ mediated the association between craving and Stroop performance. The authors concluded that low-compulsivity individuals would benefit more from top-down cognitive control interventions and high-compulsivity individuals would be better suited for stimulus-response interventions such as cue-exposure therapy or neurofeedback. The combination of cognitive task and cue-elicited paradigms allowed for researchers to pit variables against each other in order to better understand the mechanism associated with each individual's craving.

There is little empirical or conceptual focus on the role of pre-existing executive system deficits that might predispose individuals to drug craving and subsequently to drug consumption. It is clear that drug dependence contributes to neurobiological changes in the brain manifest in both executive and impulsive system dysfunction. ${ }^{12,22,33}$ What has been largely ignored, however, is whether executive system deficits increase the intensity and likelihood of craving prior to drug dependence. Executive functioning deficits observed 
in substance abusers are not independent of addiction, and likely reflect a relative imbalance between the executive and limbic systems reflected in many maladaptive behaviors (eg, overeating, gambling, risky sexual behavior). ${ }^{19,53,54}$ Longitudinal work evaluating the predictive utility of executive function measures can help elucidate the role that the executive system deficits play in predisposing individuals to craving, and subsequently, dependence. ${ }^{1}$ Longitudinal efforts might approach this problem by evaluating how measures of executive function (eg, delay discounting) and craving (eg, obsessive-compulsive drinking scale) impact future treatment gains. Existing research has failed to show consistent relationships between treatment success and craving, with some studies showing predictive utility of craving measures, ${ }^{55-57}$ whereas others do not, ${ }^{4,5}$ and the cause for disparity across studies being unknown. However, the literature linking executive functions such as delay discounting ${ }^{58-60}$ and response inhibition ${ }^{61}$ to treatment response is more consistent and might provide more sensitive measures of the underlying factors controlling executive system dysfunction in craving. A predictive model incorporating both executive function and craving may provide superior resolution, although we are unaware of studies longitudinally examining such a model.

Lastly, little empirical attention has been devoted to exploring what factors underlie the goal-directed behavior that occurs between a craving response and drug consumption. This final limitation encompasses many other limitations, including those specific to the cue-reactivity paradigm. Classical conditioning explanations of craving, for example, seem to encourage a purely autonomic conceptualization of reward processing wherein the drug cue elicits an autonomic neurobehavioral response leading to drug consumption. Additionally, neuropsychology tasks often reveal similar regions of activation as those observed in experiments on planning and goal-directed behavior. ${ }^{42}$ Drug seeking can occur without the presence of barriers (ie, automatic drug seeking); ${ }^{24}$ however, automatic drug seeking is likely mediated by an amygdaladorsal-striatal system whereas drug seeking in the face of barriers (goal-directed drug seeking) is mediated by the insula. ${ }^{16}$ What is occurring behaviorally during craving-induced "loss of control" is increasingly shown to be indicative of abnormalities in the insula region. ${ }^{15}$ The insula in part functions to process interoceptive effects of drug consumption that later serve as stimuli signaling the future likelihood of the goal-related occurrence. This might be particularly relevant when considering that fairly complex behavior must occur (controlled by executive system processes) when substance abusers experience barriers between a craving response and drug consumption. In this context, the insula is thought to contain interoceptive information that functions to process the predictive value of future rewards and facilitate goaldirected behavior. ${ }^{15}$ Such findings further reveal a need to explore performance on neuropsychological tasks, such as Go No-Go, delay discounting, and the Iowa gambling task, which have all been shown to be correlated with insula activation in substance abusers. ${ }^{16}$ Further, a two-choice prediction procedure with abstinent users by Paulus et $\mathrm{al}^{62}$ predicted continued abstinence after 1 year based on relative activation in the right insula. Evidence for the role of the insula supports the pivotal role that the executive system plays in drug-seeking behavior and also provides promising avenues for future treatment efforts.

\section{Theoretical implications}

Theories on drug craving will contribute most to the interdisciplinary literature by accomplishing two goals, ie, synthesizing and exploring issues related to both the behavioral and neurobiological characteristics of drug craving and directly informing effective treatment strategies.

One promising approach that integrates neurobiological and behavioral research and may yield novel treatment strategies is the Competing Neurobehavioral Decision Systems (CNDS) hypothesis. ${ }^{21,22,34,35,63}$ The CNDS hypothesis argues that a dysregulation in the relative balance between the executive and impulsive systems can predispose individuals to poor decision-making. ${ }^{34,64}$ This poor decision-making has been intimately linked with maladaptive responses, such as drug abuse,$^{54,65-67}$ gambling, ${ }^{20,68}$ overeating/obesity, ${ }^{19}$ and other poor health behavior. ${ }^{18}$ By contrast, healthier individuals tend to show stronger relative activation in the executive system that subserves activities such as valuing future events, response inhibition, planning, working memory, and attention. ${ }^{69}$ As described above, the executive system appears to underlie a chain of neurological events leading to a loss of control. 1,9,30,37 This chain of neurological events seems to be explained in part by interoceptive events that function as conditioned stimuli, are mediated by insula activation, and subserve goal-directed drug consumption. ${ }^{16}$

The CNDS hypothesis might be well suited to address several of the gaps in the neuroeconomic research on drug craving. The ability to identify the individual susceptibility of substance abusers via physiological and neuropsychological measurement is one example. Substance-dependent individuals have shown behavioral and neurobiological deficits in all of the executive function components listed in Table 1 (eg, valuing future events, ${ }^{21,63,64}$ working memory, ${ }^{70}$ planning, ${ }^{71}$ 
behavioral inhibition, ${ }^{72,73}$ attention, ${ }^{1,74}$ behavioral flexibility, ${ }^{71}$ and emotional regulation ${ }^{21}$ ). Craving research has revealed several key mediating networks between the executive and impulsive systems such as the fronto-accumbal network in the context of cocaine-related cues, ${ }^{75} \mathrm{a}$ frontal network including the ventral PFC and OFC downregulating the amygdala when presented with emotion-eliciting stimuli, ${ }^{76,77}$ and a prefrontal network connection to the ventral tegmental area or nucleus accumbens as a mechanism to inhibit mesolimbic dopamine neurons..$^{78,79}$ Neural networks that correlate with neuropsychological tasks can be utilized to better assess future susceptibility. Volkow et al, ${ }^{30}$ for example, found that decreases in fronto-accumbal metabolic activity were related to decreases in self-reported craving. Xue et al ${ }^{16}$ manipulated participant exposure to previous risky experiences using the recently developed Modified Cups Task ${ }^{80}$ and found that relative insular activity predicted the level of risky decisions participants made. Such findings might not only elucidate predictive markers of potential substance abusers but also provide an impetus for treatments that target such deficits (eg, neurocognitive training ${ }^{81}$ ). The study by Xue et al further demonstrated a neuroeconomic approach by creating an individualized experimental design that allowed for comparison within and across subjects.

There is currently a large imbalance between biomarkers of impulsive system craving in response to drug cues and neuropsychological tasks that elicit impulsive system activation. Table 2 demonstrates that the impulsive system has noticeably fewer hypothesized components and corresponding neuropsychological tasks. One potential explanation is that efforts to create neuropsychological tasks measuring the impulsive system have instead been utilized to measure neuroeconomic events across both systems and immediately prior to, during, and following a "loss of control". Response inhibition tasks such as Go No-Go and Stop-Signal Reaction tasks ${ }^{2,9,38,82}$ are commonly used to measure events prior to or during a loss of control and often elicit activation from both systems. Failure of the executive system to inhibit twosystem network activity derived from the impulsive system during a craving response is assumed by some researchers to be indicative of an automatic drug-craving response. ${ }^{83} \mathrm{In}$ other words, the individual user has little control over his or her behavior from the moment of craving until the presumed consumption of the drug. However, this position seems to contrast with goal-directed behavior that occurs after "loss of control" mediated by complex executive functioning processes. Goal-directed drug seeking is purported to include connectivity among regions such as the dIPFC, insula, and ACC. ${ }^{15}$ The temporal window between the "loss of control" and drug consumption might be a relevant treatment target. Specifically, can control be regained following what appears to be a maladaptive craving response? Future research might evaluate whether neurocognitive training or repetitive transcranial magnetic stimulation (rTMS)/transcranial direct current stimulation (tDCS) can strengthen self-control behavior following "loss of control." Such empirical findings should further encourage treatment efforts targeting two-system dysfunction. . $22,30,41^{2}$

Researchers using rTMS and tDCS have demonstrated that when the executive system is strengthened, craving can be reduced. ${ }^{30,84-86}$ This direct manipulation of neural activity often provides strong causal evidence that executive dysfunction likely underlies craving. Further, Boggio et a ${ }^{84}$ induced cue-elicited craving in alcohol abusers as measured

Table I Executive system components: neuropsychological task and common regions of activation

\begin{tabular}{|c|c|c|}
\hline Executive system component & Cognitive task & Common regions of activation \\
\hline Valuing future events & Delay discounting & $\begin{array}{l}\text { Dorsolateral PFC, right OFC, right ventrolateral PFC, and lateral } \\
\text { inferior parietal cortex; }{ }^{\dagger} \text { ventral striatum, medial OFC, medial PFC, } \\
\text { posterior cingulate cortex, left posterior hippocampus*,63,64,94,95 }\end{array}$ \\
\hline Working memory & O-span or n-Back & $\begin{array}{l}\text { Dorsolateral PFC, ventromedial PFC, dorsal cingulate, frontal poles, } \\
\text { frontal gyrus, posterior cingulate gyrus }{ }^{\dagger, 96}\end{array}$ \\
\hline Planning & Tower of London & Dorsolateral PFC, ventromedial PFC, and parietal cortex; ${ }^{\dagger}$ striatum $^{*, 97}$ \\
\hline Behavioral inhibition & $\begin{array}{l}\text { Stop-Signal Reaction Time } \\
\text { Task or Go-No-Go }\end{array}$ & $\begin{array}{l}\text { Right inferior frontal cortex, inferior frontal cortex, dorsolateral } \\
\text { PFC, right frontal gyrus, right medial gyrus, left cingulate, left } \\
\text { putamen, medial temporal and inferior parietal cortex }{ }^{\dagger, 82,98}\end{array}$ \\
\hline Attention & Continuous Performance Task & $\begin{array}{l}\text { Dorsolateral PFC from the posterior parietal cortex, occipital lobe, } \\
\text { lingual gyrus, and fusiform gyrus } \$, 96,99\end{array}$ \\
\hline Behavioral flexibility & Wisconsin Card-Sorting Task & Middle frontal gyrus, dorsolateral and ventromedial PFC \\
\hline Emotional regulation & lowa Gambling Task & $\begin{array}{l}\text { Medial and lateral PFC, OFC, dorsolateral PFC, and ventromedial } \\
\text { PFC; }{ }^{\dagger} \text { ACC } *, 102-104\end{array}$ \\
\hline
\end{tabular}

Notes: ${ }^{\dagger}$ Executive system regions; *impulsive system regions.

Abbreviations: ACC, anterior cingulate cortex; OFC, orbitofrontal cortex; PFC, prefrontal cortex. 
Table 2 Impulsive system components: neuropsychological task and common regions of activation

\begin{tabular}{|c|c|c|}
\hline Impulsive system component & Cognitive task & Common regions of activation \\
\hline Reward-drive and trait impulsivity & Barratt Impulsiveness Scale & Ventromedial PFC and mesolimbic regions;* $\mathrm{OFC}^{\dagger, 105,106}$ \\
\hline Reflection impulsivity & $\begin{array}{l}\text { Matching Familiar Figures Task, Information } \\
\text { Sampling Task }{ }^{108,109}\end{array}$ & Unknown at this time \\
\hline Impulsive choice & Delay discounting (immediate choice) & $\begin{array}{l}\text { Ventral striatum, medial orbitofrontal cortex, medial PFC, } \\
\text { posterior cingulate cortex, and left posterior hippocampus*63,64 }\end{array}$ \\
\hline Behavioral disinhibition & Stop-Signal Reaction Time Task or Go-No-Go & ACC;* right prefrontal cortices $s^{\dagger, 107,110}$ \\
\hline
\end{tabular}

Notes: 'Executive system regions; *impulsive system regions.

Abbreviations: ACC, anterior cingulate cortex; OFC, orbitofrontal cortex; PFC, prefrontal cortex.

by subjective craving scales and then observed how tDCS applied to the dlPFC would impact subjective craving. Cueinduced craving measured after two active tDCS sessions revealed reduced subjective craving. Equally important, craving could not be induced using the same techniques as used at the start of the study. Further support for the CNDS approach to craving can be found in the fact that rTMS to the ACC also reduces craving. ${ }^{17}$ This ability of researchers to manipulate craving levels and corresponding outcomes measures will help the evaluation of various theoretical approaches and bring researchers closer to more effective treatment options. Studies using rTMS or tDCS provide more flexibility to add repeated measures, observe immediate changes in craving, and are more cost-effective than fMRI technology. Further evaluation of the interaction of rTMS and tDCS technologies within the craving literature are needed. Relatively few existing investigations have used either technology to study craving and small sample sizes are often used. Researchers utilizing rTMS and tDCS technology, however, also often incorporate neuropsychological tasks and measures into their methodology, which strengthen the generality of their data.

Although it is difficult to target whether drug craving is a top-down or bottom-up process, research targeting executive system functioning is presently more feasible. Bickel et al, ${ }^{81}$ for example, observed that activation in the dIPFC appears to undergird both working memory and valuing future events ${ }^{87}$ by demonstrating that working memory training successfully increased the individual's ability to value future events (ie, decreased delay discounting rates). Moreover, mindfulness training, which also engages the executive system, has been shown to increase the individual's ability to value future events ${ }^{88}$ and decreases cue-elicited alcohol craving, ${ }^{89}$ suggesting a common mechanism shared by delay discounting processes and craving. Lastly, Goldstein and Volkow ${ }^{33}$ held that a dysfunctional connection between the dIPFC and nucleus accumbens reflects the interaction of a breakdown in inhibition and maladaptive reward processing, respectively. Together, these intervention strategies and exploratory studies point toward the role of the executive system, and in particular the dIPFC, as useful targets for treating drug craving.

\section{Future directions}

Future efforts to treat drug craving effectively may need to target the interactions between executive, impulsive, and mediating systems (eg, the insula and basal ganglia) in order to change goal-directed behavior. ${ }^{15}$ Currently, therapeutic approaches to craving tend to take a bottom-up approach aimed at decoupling the associative relationships between drug cues and craving responses. These Pavlovian cue exposure approaches are often context-bound, such that laboratory treatment gains fail to generalize to natural environments. ${ }^{90}$ There are more empirically studied interventions targeting executive system deficits than impulsive system deficits. Interventions targeting the executive system, therefore, may be better suited to treat maladaptive drug craving. ${ }^{1-3,23}$ Future research might target intervention strategies that build up specific executive functions related to brain regions that are activated during craving tasks (eg, dlPFC, OFC, ACC). In addition, future studies should embrace operant strategies for measuring and treating drug cravings. Researchers can utilize hypothetical purchase tasks, ${ }^{48,49,91}$ for example, to assess reinforcer demand for drug craving in order to assess the predictive and motivational value of the drug. ${ }^{30}$ In some cases, the measures of demand derived from these tasks can provide a predictive measure of relative changes in activation between two regions, in this case a fronto-accumbal network. ${ }^{30}$ Future researchers should evaluate measures of inhibition, reward valuation, and planning in relationship to goal-directed behavior following "loss of control". Specifically, methods need to be developed that target differences between successful drug seeking following a craving response (ie, maladaptive craving response) and unsuccessful drug-seeking behavior following a craving response. Such research might help to better identify the more effortful characteristics of drug seeking that can be incorporated into psychosocial interventions. ${ }^{15}$ Lastly, future work on drug craving should 
examine treatment outcomes in relation to changes in drug craving. Longitudinal studies can contribute by evaluating if executive functioning deficits observed during craving predict response to treatments. Neuroeconomic tasks (eg, delay discounting, hypothetical purchase tasks) in conjunction with cue-elicited paradigms may help researchers identify individual differences, ${ }^{36,42}$ potentially allowing for more focused treatment strategies (ie, top-down or bottom-up). Combining cue-elicited and cognitive tasks into one paradigm illustrates how a neuroeconomic approach can simultaneously provide both treatment and theoretical utility.

\section{Conclusion}

Neuroeconomic approaches are poised to address three overarching challenges in craving research: craving research must explain behavior before, during, and after a craving response; craving efforts need to provide a more fine-grained analysis of the goal-directed behavior that occurs during drug-seeking; and research must accurately reflect the complex inter-related and underlying neurobehavioral systems of craving through development of novel neuropsychological tasks.

Arguably the most prevalent barrier to craving research is the difficulty in relating a craving response to previous or future behavior. The conceptualization underlying the cuereactivity paradigm has promoted classical conditioning, ${ }^{8}$ which in turn infers the presence of a single craving response that triggers a series of complex physiological responses leading to consumption. Although many behavioral and physiological processes are occurring during the craving response, the data only summarize a sliver of time within the overall time frame of craving. Neuroeconomics can help remedy this by producing more ecologically valid measures of craving. Volkow et al, ${ }^{30}$ for example, suggest the use of a hypothetical purchase task, ${ }^{48}$ which more accurately reflects craving for the drug. The task uses parametric manipulations as opposed to a simple Likert scale (common in cue-reactivity studies) and was argued by Volkow et al to reflect the demand for the drug mediated by a fronto-accumbal network. Moreover, Gass et $\mathrm{al}^{92}$ used a personal digital assistant to assess craving in cigarette smokers in response to varenicline, allowing for frequent assessments of craving over long periods of time. Such a study could be replicated, but with additional measures using a cue-elicited paradigm (via fMRI). This would allow for a more thorough integration of craving events prior, during, and following scans. Additionally, personal digital assistants could be used to provide substance abusers with neurocognitive training that targets key executive functioning components such as delay discounting, response inhibition, and risk aversion. ${ }^{15,69}$ There are many new directions that neuroeconomic tasks can take that will broaden our understanding of the underpinnings of craving across time.

Treatment of craving must only be considered successful if it directly reduces the likelihood of drug consumption. More often than not, drug seeking is goal-directed and not automatic. ${ }^{8} 15$ From a practical standpoint, substance abusers often encounter challenges in obtaining drugs that take planning, cognitive resources, and follow-through to overcome. One barrier for researchers is access to participants when goal-directed behavior occurs. Often, participants are either aware they will receive the drug (eg, replacement therapy) or are aware they will not. In either case, there is little evidence that the behavioral and neurobiological responses measured are indicative of goal-directed drug seeking. Researchers have reliably evoked and controlled craving responses underlying automatic drug seeking; ${ }^{8}$ however, there is limited experimental evidence to date reflecting the same in goal-directed drug seeking. Available research suggests that goal-directed drug seeking in conjunction with automatic drug seeking mediate a transition from initial drug use to dependence. ${ }^{93}$ Empirical efforts must develop novel analog tasks that mirror goal-directed drug seeking in order to better reflect the complexity of underlying neural systems of craving. The task will be difficult, but may be crucial in connecting the role of craving to a reduction in drug consumption.

The success or failure of any approach to craving will in part be tied to its ability to reflect the complex inter-related systems that underlie craving. The field of neuroeconomics, by definition, holds that such systems mediate decisionmaking across intertemporal choice, risk and uncertainty, and within a social context. ${ }^{29}$ Neuroeconomic tasks often reflect two-system activation of executive functioning components. Delay discounting, for example, has been shown to activate regions across both executive and impulsive systems. ${ }^{64}$ Response inhibition has also been shown to be mediated by both systems. ${ }^{69}$ In the end, the ability of neuropsychological tasks to demonstrate the interaction between systems before, during, and after craving will allow for the methodological flexibility necessary to better understand drug craving.

In conclusion, craving reflects the relative balance between executive and impulsive systems mediated by competing processes of self-control and reward. 1,2,21,37 The present review covers: the role of craving in substance abuse and current methods; two main processes of craving, including self-control and reward-based explanations; limitations of various craving approaches and how emerging neuroeconomic approaches can fill these gaps; and, lastly, 
the theoretical implications and future directions of drugcraving research.

It is likely that craving is a top-down process where deficits in the executive system facilitate a breakdown of self-control, leading to an increased likelihood of consumption. . $^{9,23,40}$ Research on the role of drug craving in addiction indicates a need for further synthesis of neuroeconomic approaches.

\section{Disclosure}

The authors report no conflicts of interest in this work.

\section{References}

1. George $\mathrm{O}, \mathrm{Koob} \mathrm{G}$. Individual differences in prefrontal cortex function and the transition from drug use to drug dependence. Neurosci Biobehav Rev. 2010;35:232-247.

2. Volkow ND, Wang GJ, Fowler JS, Tomasi D, Telang F. Addiction: beyond dopamine reward circuitry. Proc Natl Acad Sci U SA. 2010;108: 15037-15042

3. Ma N, Liu Y, Li N, et al. Addiction related alteration in resting-state brain connectivity. Neuroimage. 2010;49:738-744.

4. Weiss RD, Griffin ML, Hufford C. Craving in hospitalized cocaine abusers as a predictor of outcome. Am J Drug Alcohol Abuse. 1995;21:289-301.

5. Walton MA, Blow FC, Bingham CR, Chermack ST. Individual and social/environmental predictors of alcohol and drug use 2 years following substance abuse treatment. Addict Behav. 2003;28: 627-642.

6. Wilson SJ, Sayette MA, Fiez JA. Prefrontal responses to drug cues: a neurocognitive analysis. Nat Neurosci. 2004;7:211-214.

7. Fatseas M, Serre F, Swendsen J, Auriacombe M. Does cue type matter for craving? Comparison of substance-specific and person-specific cues using mobile technologies. Drug Alcohol Depend. 2014;140:e57.

8. Carter BL, Tiffany ST. Meta-analysis of cue-reactivity in addiction research. Addiction. 1999;94:327-340.

9. Koob G, Volkow N. Neurocircuitry of addiction. Neuropsychopharmacology. 2010;35:217-238.

10. Skinner M, Aubin HJ. Craving's place in addiction theory: contributions of the major models. Neurosci Biobehav Rev. 2010;304:606-623.

11. Chase HW, Eickoff SB, Laird AR, Hogarth L. The neural basis of drug stimulus processing and craving: an activation likelihood estimation meta-analysis. Biol Psychiatry. 2011;70:785-793.

12. Robinson TE, Berridge KC. The neural basis of drug craving: an incentive-sensitization theory of addiction. Brain Res Rev. 1993;18: 247-291.

13. Robinson TE, Berridge KC. The incentive sensitization theory of addiction: some current issues. Philos Trans R Soc Lond B Biol Sci. 2008;363:3137-3146.

14. Koob GF. Drug addiction: the yin and yang of hedonic homeostasis. Neuron. 1996;16:893-896.

15. Naqvi NH, Gaznick N, Tranel D, Bechara A. The insula: a critical neural substrate for craving and drug seeking under conflict and risk. Ann NY Acad Sci. 2014;1316:53-70.

16. Xue G, Lu Z, Levin IP, Bechara A. The impact of prior risk experiences on subsequent risky decision-making: the role of the insula. Neuroimage. 2010;50:709-716.

17. De Ridder D, Vanneste S, Kovacs S, Sunaert S, Dom G. Transient alcohol craving suppression by rTMS of dorsal anterior cingulate: an fMRI and LORETA EEG study. Neurosci Lett. 2011;496:5-10.

18. Bickel WK, Jarmolowicz DP, Mueller ET, Koffarnus MN, Gatchalian KM. Excessive discounting of delayed reinforcers as a trans-disease process contributing to addiction and other disease-related vulnerabilities: emerging evidence. Pharmacol Ther. 2012;134:287-297.
19. Jarmolowicz DP, Cherry JB, Reed DD, et al. Robust relation between temporal discounting rates and body mass. Appetite. 2014;78: 63-67.

20. Dixon MR, Marley J, Jacobs EA. Delay discounting by pathological gamblers. J Appl Behav Anal. 2003;36:449-458.

21. Bechara A. Decision making, impulse control and loss of willpower to resist drugs: a neurocognitive perspective. Nat Neurosci. 2005;8: $1458-1463$

22. Jentsch JD, Taylor JR. Impulsivity resulting from frontostriatal dysfunction in drug abuse: implications for the control of behavior by reward-related stimuli. Psychopharmacology. 1999;146:373-390.

23. Yang Z, Xie J, Shao YC, et al. Dynamic neural responses to cuereactivity paradigms in heroin-dependent users: an fMRI study. Hum Brain Mapp. 2009;30:766-775.

24. Tiffany ST, Conklin CA. A cognitive processing model of alcohol craving and compulsive alcohol use. Addiction. 2000;95 Suppl 2: S145-S153.

25. Tiffany ST. The role of cognitive factors in reactivity to drug cues. In: Drummond DC, Tiffany ST, Glautier S, Remington B, editors. Addictive Behaviour: Cue Exposure Theory And Practice. Chichester, UK: John Wiley \& Sons; 1995.

26. Tiffany ST. A cognitive model of drug urges and drug-use behavior: the role of automatic and non-automatic processes. Psychol Rev. 1990;97:147-168.

27. Baker TB, Cannon DS, Tiffany ST, Gino A. Cardiac response as an index of the effect of aversion therapy. Behav Res Ther. 1984;22: 403-411.

28. Kahneman D, Tversky A. Prospect theory: an analysis of decision under risk. Econometrica. 1979;47:263-291.

29. Loewenstein G, Rick S, Cohen JD. Neuroeconomics. Annu Rev Psychol. 2008;59:647-672.

30. Volkow ND, Fowler JS, Wang GJ, et al. Cognitive control of drug craving inhibits brain reward regions in cocaine abusers. Neuroimage. 2010;49:2536-2543.

31. Robinson TE, Berridge KC. Addiction. Аnnu Rev Psychol. 2000;54: $25-53$.

32. Langleben DD, Ruparel K, Busch-Winokur S, et al. Acute effect of methadone maintenance dose on brain fMRI response to heroin-related cues. Am J Psychiatry. 2008; 165:390.

33. Goldstein RZ, Volkow ND. Drug addiction and its underlying neurobiological basis: neuroimaging evidence for the involvement of the frontal cortex. Am J Psychiatry. 2002;159:1642-1652.

34. Bickel WK, Miller ML, Yi R, Kowal BP, Lindquist DM, Pitcock JA. Behavioral and neuroeconomics of drug addiction: competing neural systems and temporal discounting processes. Drug Alcohol Depend. 2007;90S:S85-S91.

35. Koffarnus MN, Jarmolowicz DP, Mueller ET, Bickel WK. Changing discounting in light of the competing neurobehavioral decision systems theory. J Exp Anal Behav. 2013;99:32-57.

36. Lerman C, Gu H, Loughead J, Ruparel K, Yang Y, Stein EA. Large-scale brain network coupling predicts acute nicotine abstinence effects on craving and cognitive function. JAMA Psychiatry. 2014;71:523-530.

37. Baler RD, Volkow N. Drug addiction: the neurobiology of disrupted self-control. Trends Mol Med. 2006;12:559-566.

38. Aron AR. From reactive to proactive and selective control: developing a richer model for stopping inappropriate responses. Biol Psychiatry. 2011;69:e55-e68.

39. Eagle DM, Baunez C, Hutcheson DM, Lehmann O, Shah AP, Robbins TW. Stop-signal reaction-time task performance: role of prefrontal cortex and subthalamic nucleus. Cereb Cortex. 2008;18: 178-188.

40. Liu J, Qin W, Yuan K, et al. Interaction between dysfunctional connectivity at rest and heroin cues-induced brain responses in male abstinent heroin-dependent individuals. PLoS One. 2011;6:1-11.

41. Fu L, Bi G, Zou Z, et al. Impaired response inhibition function in abstinent heroin dependents: an fMRI study. Neurosci Lett. 2008;438: $322-326$. 
42. Verdejo-Garcia A, Bechara A, Recknor EC, Perez-Garcia M. Executive dysfunction in substance dependent individuals during drug use and abstinence: an examination of the behavioral, cognitive and emotional correlates of addiction. J Int Neuropsychol Soc. 2006;12:405-415.

43. Kalivas P, O’Brien CP. Drug addiction as a pathology of staged neuroplasticity. Neuropsychopharmacology. 2008;33:168-180.

44. Everitt BJ, Wolf ME. Psychomotor stimulant addiction: a neural systems perspective. J Neurosci. 2002;22:3312-3320.

45. Sidman M. Tactics of Scientific Research: Evaluating Experimental Data in Psychology. New York, NY, USA: Basic Books; 1960.

46. LaConte S. Decoding fMRI brain states in real-time. Neuroimage. 2011;56:440-454.

47. Canterberry M, Hanlon C, Hartwell K, et al. Sustained reduction of nicotine craving with real-time neurofeedback: exploring the role of severity of dependence. Nicotine Tob Res. 2013;15:2120-2124.

48. Jacobs EA, Bickel WK. Modeling drug consumption in the clinic via simulation procedures: demand for heroin and cigarettes in opioid-dependent outpatients. Exp Clin Psychopharmacol. 1999;7: 412-426.

49. MacKillop J, Brown C, Stojek M, Murphy C, Sweet L, Niaura R. Behavioral economic analysis of withdrawal- and cue-elicited craving for tobacco: an initial investigation. Nicotine Tob Res. 2012;14:1426-1434.

50. Bickel WK, Jarmolowicz DP, MacKillop J, et al. The behavioral economics of reinforcement pathologies. In: Shaffer HJ, editor. Addiction Syndrome Handbook. Washington, DC, USA: American Psychological Association; 2013.

51. Verdejo-García A, Lubman DI, Schwerk A, et al. Effect of craving induction on inhibitory control in opiate dependence. Psychopharmacology. 2012;219:519-526.

52. Franken IH, Hendriks VM, van den Brink W. Initial validation of two opiate craving questionnaires: the Obsessive Compulsive Drug Use Scale and the Desires for Drug Questionnaire. Addict Behav. 2002;27: 675-685.

53. Jarmolowicz DP, Bickel WK, Gatchalian KM. Alcohol-dependent individuals discount sex at higher rates than controls. Drug Alcohol Depend. 2013;131:320-323.

54. Petry NM, Casarella R. Excessive discounting of delayed rewards in substance abusers with gambling problems. Drug Alcohol Depend. 1999;56:25-32.

55. Marhe R, Luijten M, van de Wetering BJ, Smits M, Franken IH Individual differences in anterior cingulate activation associated with attentional bias predict cocaine use after treatment. Neuropsychopharmacology. 2013;38:1085-1093.

56. Paliwal P, Hyman SM, Sinha R. Craving predicts time to cocaine relapse: further validation of the Now and Brief versions of the cocaine craving questionnaire. Drug Alcohol Depend. 2008;93:252-259.

57. Rosenberg H. Clinical and laboratory assessment of the subjective experience of drug craving. Clin Psychol Rev. 2009;29:519-534.

58. Stanger C, Elton A, Ryan SR, James AG, Budney AJ, Kilts CD. Neuroeconomics and adolescent substance abuse: individual differences in neural networks and delay discounting. J Am Acad Child Adolesc Psychiatry. 2013;52:747-755.

59. Washio Y, Higgins ST, Heil SH, et al. Delay discounting is associated with treatment response among cocaine-dependent outpatients. Exp Clin Psychopharmacol. 2011;19:243-248.

60. Yoon JH, Higgins ST, Heil SH, Sugarbaker RJ, Thomas CS, Badger GJ. Delay discounting predicts postpartum relapse to cigarette smoking among pregnant women. Exp Clin Psychopharmacol. 2007;15: 176-186.

61. Goldenberg D, Thayer R, Migliorini R, Simmons AN, Tapert SF. Adolescents' fMRI activation to a response inhibition task predicts future substance use. Addict Behav. 2013;38:1435-1441.

62. Paulus MP, Tapert SF, Schuckit MA. Neural activation patterns of methamphetamine-dependent subjects during decision making predict relapse. Arch Gen Psychiatry. 2005;62:761-768.

63. McClure SM, Ericson KM, Laibson DI, Loewenstein G, Cohen JD. Time discounting for primary rewards. J Neurosci. 2007;27:5796-5804.
64. McClure SM, Laibson DI, Loewenstein G, Cohen JD. Separate neural systems value immediate and delayed monetary rewards. Science. 2004;306:503-507.

65. Bjork JM, Hommer DW, Grant SJ, Danube C. Impulsivity in abstinent alcohol-dependent patients: relation to control subjects and type 1-/type 2 like traits. Alcohol. 2004;34:133-150.

66. Allen TJ, Moeller FG, Rhoades HM, Cherek DR. Impulsivity and history of drug dependence. Drug Alcohol Depend. 1998;50:137-145.

67. Petry NM, Bickel WK, Arnett M. Shortened time horizons and insensitivity to future consequences in heroin addicts. Addiction. 1998;93:729-738.

68. Mackillop J, Anderson EJ, Castelda BA, Mattson RE, Donovick PJ. Convergent validity of measures of cognitive distortions, impulsivity, and time perspective with pathological gambling. Psychol Addict Behav. 2006;20:75-79.

69. Bickel WK, Jarmolowicz DP, Mueller ET, Gatchalian KM, McClure SM. Are executive function and impulsivity antipodes? A conceptual reconstruction with special reference to addiction. Psychopharmacology. 2012;221:361-387.

70. Baddeley A. Working memory: looking back and looking forward. Nat Rev Neurosci. 2003;4:829-839.

71. Robbins TW. Dissociating executive functions of the prefrontal cortex. Philos Trans R Soc Lond B Biol Sci. 1996;351:1463-1471.

72. Barkley RA. Behavioral inhibition, sustained attention, and executive functions: constructing a unifying theory of ADHD. Psychol Bull. 1997;121:65-94.

73. Barkley RA. Adolescents with attention-deficit/hyperactivity disorder: an overview of empirically based treatments. $J$ Psychiatr Pract. 2004;10:39-56.

74. Garavan H, Hester R. The role of cognitive control in cocaine dependence. Neuropsychol Rev. 2007;17:337-345.

75. Volkow ND, Wang GJ, Tomasi D, et al. Methylphenidate attenuates limbic brain inhibition after cocaine-cues exposure in cocaine abusers (brain activity during craving). PLoS One. 2010;5:e11509.

76. Urry HL, van Reekum CM, Johnstone T, et al. Amygdala and ventromedial prefrontal cortex are inversely coupled during regulation of negative affect and predict the diurnal pattern of cortisol secretion among older adults. $J$ Neurosci. 2006;26:4415-4425.

77. Johnstone T, van Reekum CM, Urry HL, Kalin NH, Davidson RJ. Failure to regulate: counterproductive recruitment of top-down prefrontal-subcortical circuitry in major depression. J Neurosci. 2007;27:8877-8884

78. Carr DB, Sesack SR. Projections from the rat prefrontal cortex to the ventral tegmental area: target specificity in the synaptic associations with mesoaccumbens and mesocortical neurons. J Neurosci. 2000;20: 3864-3873.

79. Sesack SR, Pickel VM. Prefrontal cortical efferents in the rat synapse on bunlabeled neuronal targets of catecholamine terminals in the nucleus accumbens septi and on dopamine neurons in the ventral tegmental area. J Comp Neurol. 1992;320:145-160.

80. Levin I, Weller J, Pederson A, Harshman L. Age-related differences in adaptive decision making: sensitivity to expected value in risky choice. Judgm Decis Mak. 2007;2:225-233.

81. Bickel WK, Yi R, Landes RD, Hill PF, Baxter C. Remember the future: working memory training decreases delay discounting among stimulant addicts. Biol Psychiatry. 2011;69:260-265.

82. Aron AR, Robbins TW, Poldrack R. Inhibition and the right inferior frontal cortex. Trends Cogn Sci. 2004;8:170-177.

83. Conklin CA, Tiffany ST. Applying extinction research and theory to cue-exposure addiction treatments. Addiction. 2002;97:155-167.

84. Boggio PS, Sultani N, Fecteau S, et al. Prefrontal cortex modulation using transcranial DC stimulation reduces alcohol craving: a doubleblind, sham-controlled study. Drug Alcohol Depend. 2008;92:55-60.

85. Amiaz R, Levy D, Vainiger D, Grunhaus L, Zangen A. Repeated highfrequency transcranial magnetic stimulation over the dorsolateral prefrontal cortex reduces cigarette craving and consumption. Addiction . 2008;104:653-660. 
86. Li X, Hartwell KJ, Owens M, et al. Repetitive transcranial magnetic stimulation of the dorsolateral prefrontal cortex reduces nicotine cue craving. Biol Psychiatry. 2013;73:714-720.

87. Wesley MJ, Bickel WK. Remember the future II: meta-analyses and functional overlap of working memory and delay discounting. Biol Psychiatry. 2014;75:435-448.

88. Morrison KL, Madden GJ, Odum AL, Friedel JE, Twohig MP. Altering impulsive decision making with an acceptance-based procedure. Behav Ther. 2014;45:630-639.

89. Garland EL, Gaylord SA, Boettiger CA, Howard MO. Mindfulness training modifies cognitive, affective, and physiological mechanisms implicated in alcohol dependence: results of a randomized controlled pilot trial. J Psychoactive Drugs. 2010;42:177-192.

90. Havermans RC, Jansen AT. Increasing the efficacy of cue exposure treatment in preventing relapse of addictive behavior. Addict Behav. 2003;28:989-994.

91. Murphy JG, MacKillop J. Relative reinforcing efficacy of alcohol among college student drinkers. Exp Clin Psychopharmacol. 2006;14: 219-227.

92. Gass JC, Wray JM, Hawk LW, Mahoney MC, Tiffany ST. Impact of varenicline on cue-specific craving assessed in the natural environment among treatment-seeking smokers. Psychopharmacology. 2012;223: 107-116.

93. Hogarth L. The role of impulsivity in the aetiology of drug dependence: reward sensitivity versus automaticity. Psychopharmacology. 2011; 215:567-580

94. Kable JW, Glimcher PW. The neural correlates of subjectiveb value during intertemporal choice. Nat Neurosci. 2007;10:1625-1633.

95. Kable JW, Glimcher PW. An "as soon as possible" effect in human intertemporal decision making: behavioral evidence and neural mechanisms. J Neurophysiol. 2010;103:2513-2531.

96. de Fockert JW, Rees G, Frith CD, Lavie N. The role of working memory in visual selective attention. Science. 2001;291:1803-1806.

97. Shallice T, Burgess PW. Deficits in strategy application following frontal lobe damage in man. Brain. 1991;114:727-741.

98. Norman AL, Pulido C, Squeglia LM, Spadoni AD, Paulus MP, Tapert SF. Neural activation during inhibition predicts initiation of substance use in adolescence. Drug Alcohol Depend. 2011;119:216-223.
99. Miller EK, Cohen JD. An integrative theory of prefrontal cortex function. Annu Rev Neurosci. 2001;24:167-202.

100. Bechara A, Damasio AR, Damasio H, Anderson SW. Insensitivity to future consequences following damage to human prefrontal cortex. Cognition. 1994;50:7-15.

101. Bechara A, Damasio H, Tranel D, Damasio AR. Deciding advantageously before knowing the advantageous strategy. Science. 1997;275:1293-1295.

102. Boggio PS, Campanha C, Valasek CA, Fecteau S, Pascual-Leone A, Fregni F. Modulation of decision-making in a gambling task in older adults with transcranial direct current stimulation. Eur J Neurosci. 2010;31:593-597.

103. Fukui H, Murai T, Fukuyama H, Hayashi T, Hanakawa T. Functional activity related to risk anticipation during performance of the Iowa Gambling Task. Neuroimage. 2005;24:253-259.

104. Bechara A, Damasio H, Damasio AR. Role of the amygdala in decisionmaking. Ann NY Acad Sci. 2003;985:356-369.

105. Sripada CS, Gonzalez R, Luan Phan K, Liberzon I. The neural correlates of intertemporal decision-making: contributions of subjective value, stimulus type, and trait impulsivity. Hum Brain Mapp. 2011;32:1637-1648.

106. Gullo M, Dawe S. Impulsivity and adolescent substance use: rashly dismissed as "all-bad"? Neurosci Biobehav Rev. 2008;32: 1507-1518.

107. Hester RK, Garavan H. Executive dysfunction in cocaine addiction: evidence for discordant frontal, cingulated, and cerebellar activity. J Neurosci. 2004;24:11017-11022.

108. Kagan J. Reflection-impulsivity: the generality and dynamics of conceptual tempo. J Abnorm Psychol. 1966;71(1):17-24.

109. Clark L, Robbins TW, Ersche KD, Sahakian BJ. Reflection impulsivity in current and former substance users. Biol Psychiatry. 2006;60(5):515-522.

110. Monterosso JR, Aron AR, Cordova X, Xu J, London ED. Deficits in response inhibition associated with chronic methamphetamine abuse. Drug Alcohol Depend. 2005;79:273-277.
Neuroscience and Neuroeconomics

\section{Publish your work in this journal}

Neuroscience and Neuroeconomics is an international, peer-reviewed, open access journal focusing on the identification of brain structures and measurement of neural activity related to behavior, behavioral predictions, and decision making in health and disease. The manuscript management system is completely online and includes a very quick and

\section{Dovepress}

fair peer-review system. Visit http://www.dovepress.com/testimonials php to read real quotes from published authors. 